\title{
Genomic signatures of host adaptation in group B Salmonella enterica ST416/ST417 from harbour porpoises
}

\author{
Arnar K. S. Sandholt ${ }^{1}$, Aleksija Neimanis ${ }^{2}$, Anna Roos ${ }^{3}$, Jenny Eriksson ${ }^{1}$ and Robert Söderlund ${ }^{1 *}$ (D)
}

\begin{abstract}
A type of monophasic group B Salmonella enterica with the antigenic formula 4,12:a:- ("Fulica-like") has been described as associated with harbour porpoises (Phocoena phocoena), most frequently recovered from lung samples. In the present study, lung tissue samples from 47 porpoises found along the Swedish coast or as bycatch in fishing nets were analysed, two of which were positive for S. enterica. Pneumonia due to the infection was considered the likely cause of death for one of the two animals. The recovered isolates were whole genome sequenced and found to belong to sequence type (ST) 416 and to be closely related to ST416/ST417 porpoise isolates from UK waters as determined by core-genome MLST. Serovars Bispebjerg, Fulica and Abortusequi were identified as distantly related to the porpoise isolates, but no close relatives from other host species were found. All ST416/417 isolates had extensive loss of function mutations in key Salmonella pathogenicity islands, but carried accessory genetic elements associated with extraintestinal infection such as iron uptake systems. Gene ontology and pathway analysis revealed reduced secondary metabolic capabilities and loss of function in terms of signalling and response to environmental cues, consistent with adaptation for the extraintestinal niche. A classification system based on machine learning identified ST416/417 as more invasive than classical gastrointestinal serovars. Genome analysis results are thus consistent with ST416/417 as a host-adapted and extraintestinal clonal population of S. enterica, which while found in porpoises without associated pathology can also cause severe opportunistic infections.
\end{abstract}

Keywords: Pangenome analysis, cgMLST, salmonellosis, marine mammals, cetaceans, host bias, molecular epidemiology, extraintestinal infection, porpoise, Phocoena

\section{Introduction}

The harbour porpoise (Phocoena phocoena) is a species of small cetaceans that inhabit coastal waters of the northern hemisphere. While it is abundant in the North Atlantic and the North Pacific, the Baltic Sea and Black Sea populations are critically endangered [1]. Because of their near-shore habitat, harbour porpoises are vulnerable to anthropogenic environmental disturbance

*Correspondence: robert.soderlund@sva.se

${ }^{1}$ Department of Microbiology, National Veterinary Institute, Uppsala, Sweden

Full list of author information is available at the end of the article including chemical and noise pollution and prey depletion as well as entanglement in fishing nets, especially in semi-enclosed seas such as the Baltic [1]. Such environmental stressors can weaken the immune system and leave animals more vulnerable to infection [2].

Harbour porpoises in Swedish waters can be classified into three populations: The North Sea, Belt Sea and Baltic Proper populations. Animals that are found dead are collected and examined to identify causes of death and other threats [3]. In a study of 98 stranded porpoises collected from 2008 to 2019, infectious disease was the second most common cause of death (17\%) after bycatch and probable bycatch (25\%). Infectious diseases included 
several cases of bacterial pneumonia in the presence of nematodes [3]. This is consistent with reports of a high frequency of pneumonia associated with parasites, bacterial infection, or both, among porpoises from the North Atlantic examined post-mortem [4-8].

A particular type of monophasic group B Salmonella enterica subsp. enterica, most often of sequence type (ST) 416 and with the antigenic formula 4,12:a:- ("Fulicalike") has been described as exclusively associated with harbour porpoises in Scotland and England $[9,10]$. This type of Salmonella occurs more frequently in lung samples than other tissues or faeces [11], and has also been recovered from porpoise lung worms [12] suggesting a possible mode of transmission. As low-grade Salmonella infections in the lungs appear to be prevalent among porpoises, it has been hypothesized that the infection becomes severe or life-threatening primarily in weakened individuals [11]. While most S. enterica are host generalists and cause self-limiting gastroenteritis in infected animals or humans, host adapted Salmonella serovars tend to infect one or a few host species, have a higher likelihood of causing extraintestinal infection, and more frequently establish a chronic carrier state in otherwise healthy infected animals, typical examples being $S$. Dublin in cattle, $S$. Choleraesuis in pigs and $S$. Gallinarum in poultry [13]. The evolution of a host adapted lineage of Salmonella from generalist ancestors appears to involve extensive degradation of genes not needed in the specialist niche with a corresponding loss of metabolic capability, as well as acquisition of mobile genetic elements with genes providing new traits [14-18]. Host and niche adaptation of the proposed porpoise-associated strains of Salmonella should therefore be evident in their genome sequences. In the present study we report the occurrence of S. enterica ST416 in lung samples from Swedish harbour porpoises and show extensive genomic evolution in these strains consistent with host adaptation and an increased capacity for causing extraintestinal infection.

\section{Materials and methods}

Animals, samples, and bacterial isolates

In Sweden, porpoises and several other mammalian and avian species of conservation interest are state property ("statens vilt" or "wildlife of the state") and must be reported to the authorities if they are found dead. From 2008 to 2020, 142 porpoises found along the Swedish coast or incidentally killed as bycatch in fishing nets have been collected for necropsy examination within a collaborative program delivered by the Swedish National Veterinary Institute (SVA) and the Swedish Museum of Natural History. Almost all animals were stored frozen before investigation. Porpoises were examined following standardized protocols [19] to determine cause of death, investigate any abnormalities and collect tissues and data for other studies and archives [3]. Representative pieces of lung were fixed in $10 \%$ neutrally buffered formalin and processed and embedded in paraffin for microscopic examination. Sections $(3-4 \mu \mathrm{m})$ were stained using Mayer's hematoxylin and eosin [20]. Prior to 2018, any lesions consistent with possible bacterial infection were submitted for aerobic bacterial culture $(n=9$ animals, of which lung was cultured in 5 animals) as described below. Since 2018, lung tissue from all porpoises that were not more than moderately decomposed were submitted for aerobic bacterial culture only $(n=21)$, for selective culture of Salmonella sp. $(n=15)$ or for both $(n=8)$. In total, lung tissue was cultured from 47 porpoises in this study. All bacteriology was performed by the Department of Microbiology, SVA. For aerobic bacterial culture samples were inoculated onto blood agar plates containing 5\% horse blood and bromocresol purple lactose agar plates and held at $37{ }^{\circ} \mathrm{C}$ under aerobic conditions. In parallel, horse blood agar plates were grown at $37{ }^{\circ} \mathrm{C}$ in a $5 \% \pm 1 \% \mathrm{CO}_{2}$ incubator. Plates were inspected for growth at 24 and $48 \mathrm{~h}$ after inoculation. For selective culture, ISO 65791:2017 was followed with samples enriched in buffered peptone water and grown on modified semi-solid Rappaport-Vassiliadis (MSRV) agar, horse blood agar, brilliant green (BG) agar and xylose lysine deoxycholate (XLD) agar. Suspected Salmonella colonies were confirmed to species level by either real-time PCR [21] (in 2017) or by MALDI-TOF MS on a Bruker Biotyper instrument (in 2020) according to manufacturer instructions. Serotyping was performed with biochemical testing and slide agglutination, also according to ISO 6579-1:2017.

\section{Sequencing and reference data}

Libraries were generated from 17-VLT002652 with a Nextera XT kit and sequencing performed on an Illumina MiSeq instrument as $2 \times 250$ bp paired-end. Additional long read data was generated using an Oxford Nanopore MinION sequencing device run with a R9.4.1 flow cell and a library created using the Rapid PCR barcoding kit. Sequencing of the second isolate 20-VLT001389 was performed by Illumina technology in the same way but as $250+60$ bp paired-end due to technical issues with the reverse read. All three sequence datasets were a minimum of $100 \times$. Short read data from 59 close relatives of the Swedish porpoise strains were identified by core genome multi-locus sequence typing (cgMLST) via Enterobase [22], together forming group 4372 at the hierarchical clustering level $\mathrm{HC} 2000$ as defined by the HierCC algorithm. Short read data from these isolates were downloaded from the European Nucleotide Archive for inclusion in downstream analysis. Short read data was quality checked using FastQC v0.11.8 and trimmed 
using Trimmomatic v0.39, with a sliding window of $4 \mathrm{bp}$ and quality threshold of 20 . Long read data was quality checked using FastQC and NanoPlot v1.32.1 [23] and filtered using Filtlong v0.2.0 keeping the best $900 \mathrm{Mbp}$ of reads. The $S$. enterica type strain LT2 (S. Typhimurium) genome, GenBank NC_003197, was used as reference for read mapping, annotation, and analysis of Salmonella pathogenicity islands.

\section{Data analysis and visualization}

Bowtie2 v2.3.5.1 was used to map short read data to the LT2 reference genome, and Samtools v1.9 was used for single nucleotide polymorphism (SNP) calling. The resulting VCF files were filtered using in-house $\mathrm{R}$ scripts (Additional file 4) and quality checked using the VCFR package v1.8.0. SNPEff v4.3 [24] was used to evaluate the impact of each SNP found. Gene Ontology (GO) categories impacted by the mutations were found using the Panther GO Enrichment tool [25] and the KEGG Mapper tool [26]. A machine learning algorithm developed by Wheeler et al. [27] was applied to read data from all 61 strains to assess the invasiveness of each and how it compared to known enteric and invasive Salmonella serovars. Unicycler v0.4.8 [28] with default settings was used to create a hybrid assembly for 17-VLT002652, and assemblies from short reads only for all other isolates. Read data were downsampled to $100 \times$ before assembly. Assemblies were inspected using Bandage [29] when necessary. Annotation of assemblies was performed using Prokka v1.14.5 [30] using the S. enterica type strain $S$. Typhimurium LT2 as a reference and default settings. Roary v3.13.0 [31] was run on the annotated assemblies, with a $90 \%$ identity cutoff and otherwise default settings, to identify the core and pan genomes. Scoary v1.6.16 [32] was run on the results to identify genes that were significantly more frequently observed in porpoise isolates compared to the reference isolate genomes. Structural information for the interpretation of results was accessed via the hybrid assembly of 17-VLT002652. 7-gene MLST and cgMLST were performed via Enterobase [22]. A minimum-spanning tree based on cgMLST data was generated with GrapeTree via Enterobase. Boxplots and gene maps were drawn in $\mathrm{R} 2.5 .0$ [33] with the gggenes package [34].

\section{Results}

\section{Necropsy findings associated with Salmonella infection}

The first Salmonella isolate in the present study (17VLT002652) was recovered from a porpoise found stranded in Varberg municipality (N 57 $7.1^{\prime}$, E 12 $11.5^{\prime}$ ) on the Swedish west coast in July 2017. This mature, 18-year-old male was in very poor nutritional condition and suffered from severe bronchopneumonia in which
$50-60 \%$ of the lung tissue was necrotic and inflamed (Figure 1). There was a concurrent, moderate to heavy nematode infection in the airways with few parasites in the heart and pulmonary vessels. Microscopically, large areas of acute pyogranulomatous inflammation and necrosis consistent with acute bacterial pneumonia were seen and within some areas, bacteria could also be identified (Figure 2). Parasites were not associated with these lesions. Given the pathological changes seen associated with bacteria and the abundant growth of Salmonella from lung tissue, pneumonia caused by Salmonella infection was determined to be the cause of death. The recovered Salmonella isolate could not be serotyped and was biochemically atypical (lysine decarboxylase negative). The second isolate (20-VLT001389) was cultured from an animal found dead in February 2020 in Helsingborg (N $56^{\circ} 2.7^{\prime}, \mathrm{E} 12^{\circ} 41.2^{\prime}$ ), further south on the west coast. This immature female porpoise was in good nutritional condition and deemed to be in good health. Linear marks on the head and extremities were consistent with net marks and death was attributed to incidental capture in fishing gear. This animal had a moderate burden of nematodes in the airways and scattered areas of caseous inflammation in the lung tissue. Microscopically, focal areas of chronic eosinophilic and granulomatous pneumonia typical of parasitic infection were seen associated with nematodes. No lesions consistent with bacterial pneumonia

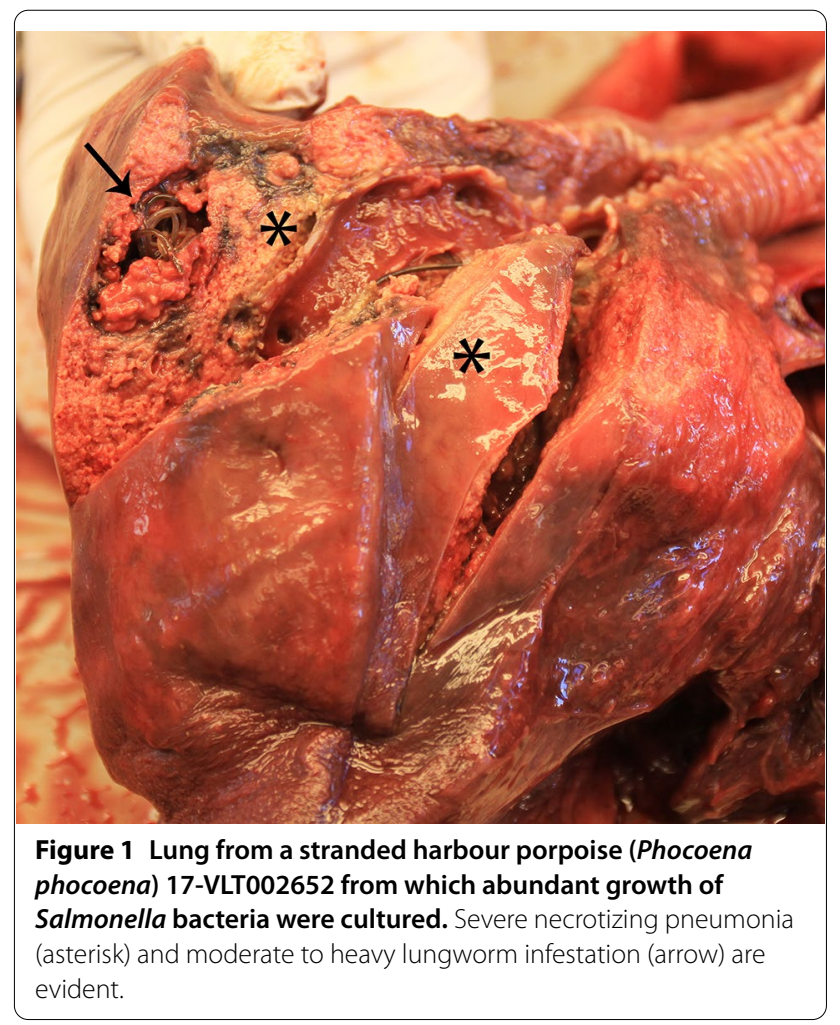




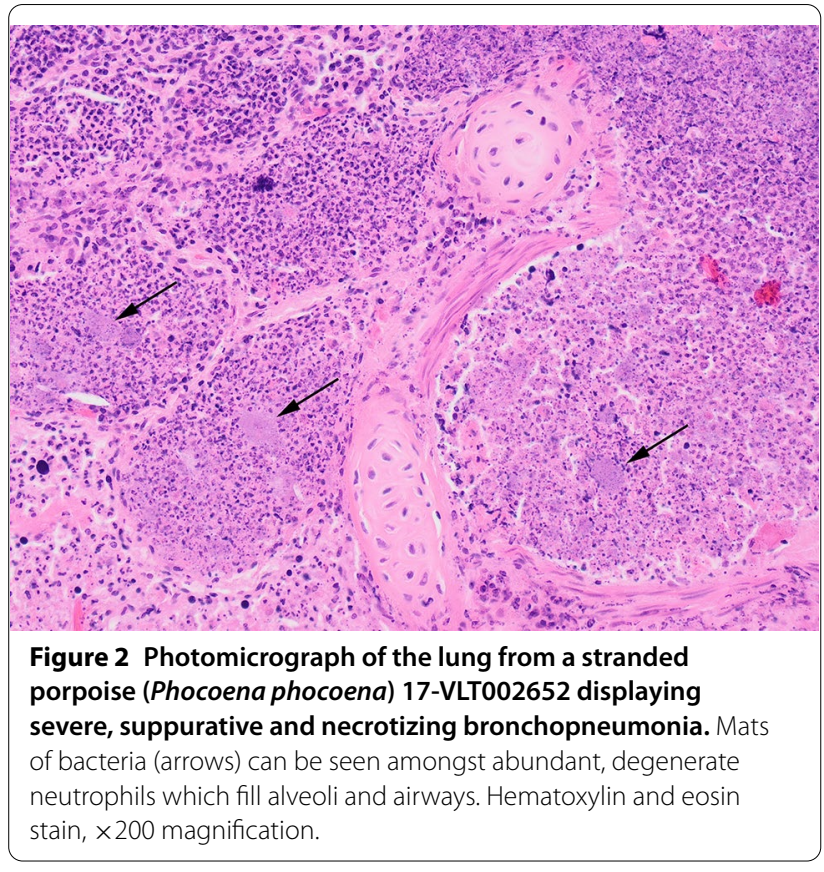

were observed and only weak growth of Salmonella was observed following culture of the lung. The presence of Salmonella bacteria in the lungs of this animal was not associated with any pathology. This second isolate was also lysine decarboxylase negative and was serotyped as 4:a:--

\section{Core-genome MLST comparison of porpoise Salmonella and identification of close relatives}

Both Swedish porpoise isolates were ST416. Phylogenetic analysis based on cgMLST indicated that the Swedish isolates most closely matched 17 "Fulica-like" or "Fulicalike rough" ST416 or ST417 isolates in Enterobase, of which 16 were from harbour porpoises in Scotland and 1 was from Scotland but lacked metadata. The Swedish isolates were most similar to each other but clustered closely with the main group of Scottish isolates. Isolates of serovars Bispebjerg $(n=23)$, Fulica $(n=2)$, Abortusequi $(n=10)$ and of unknown serovars $(n=7)$ were identified as the best matches from sources other than porpoises based on belonging to the cgMLST group 4372 at the hierarchical clustering level HC2000 but were far less closely related (Figure 3 ). Most included Bispebjerg isolates were from humans $(n=12)$, with additional isolates from turtles $(n=4)$, food $(n=2)$, a falcon $(n=1)$ or unspecified sources. The only two isolates of Fulica, both isolated from humans, clustered together but as part of the Bispebjerg cgMLST cluster. The Abortusequi isolates were from horses $(n=2)$ or unspecified sources. The isolates without specified serotypes were from humans

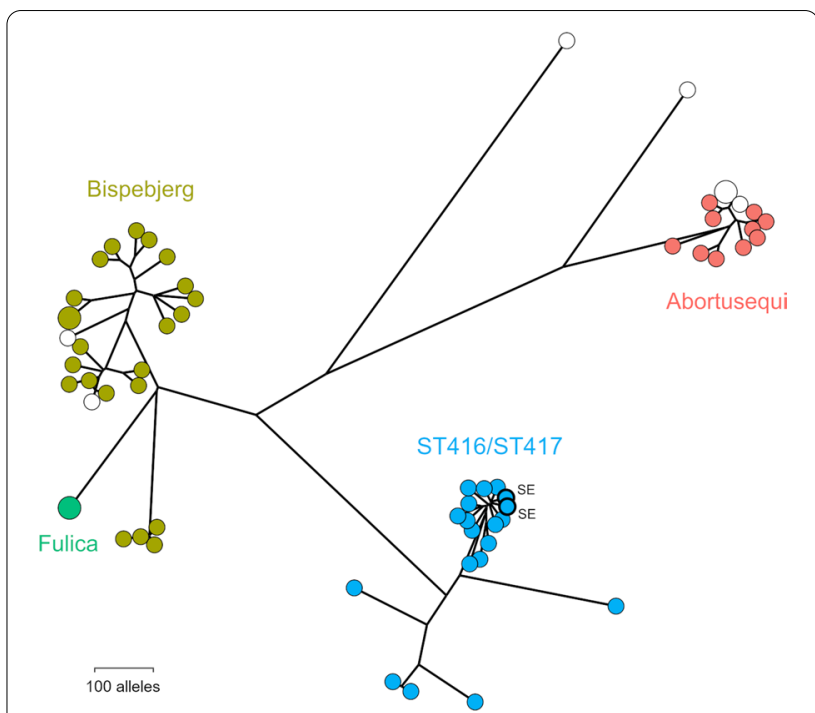

Figure 3 Phylogenetic relationship between harbour porpoise isolates of S. enterica ST416/ST417 and their nearest neighbours as determined by cgMLST. The closest relatives identified include isolates identified as serovars Bispebjerg, Fulica and Abortusequi. Swedish isolates sequenced in the present study are labelled SE. White circles indicate isolates for which no serotypes were reported.

$(n=2)$, palm nuts $(n=2)$, a donkey $(n=1)$ or unspecified sources.

Alterations in major virulence associated genomic regions The $\operatorname{spv} R, A, B, C, D$ genes forming the signature locus of the Salmonella virulence plasmids [35] were absent in all ST416/417 isolates. The spv locus was present in most $S$. Abortusequi isolates, and a single isolate with no serovar assigned, while absent from the remaining genomes analysed. Major structural differences were observed between ST416/417 isolate pathogenicity islands SPI1-5 and those of the related isolates (Figure 4, Additional file 1). SPI-1 was found to be partially deleted with hilA which is a transcriptional regulator necessary for SPI-1 expression [36] missing together with $i a g B$ and the translocated effector proteins $\operatorname{spt} P$, sipA and $\operatorname{sipD}$. The majority of SPI-2 was also absent, leaving only $s s r B$ and a segment containing $s s a U, T, S, R$. SPI-3 was completely absent together with a $\sim 15 \mathrm{kbp}$ downstream genomic region present in LT2. SPI-4, which encodes a T1SS and a single large secreted adhesin, was present in the 17-VLT002652 genome, but both the adhesin gene (siiE, annotated as a hypothetical protein with locus tag STM4261 in the LT2 genome) and an essential gene for the T1SS (siiF, locus tag STM4262) were disrupted by ISelement insertions in coding regions. SPI- 5 was also partially absent, with only $\operatorname{sop} B$ and pip $A, B, C$ remaining. A short fragment consistent with a partial SPI-5 pipD gene 


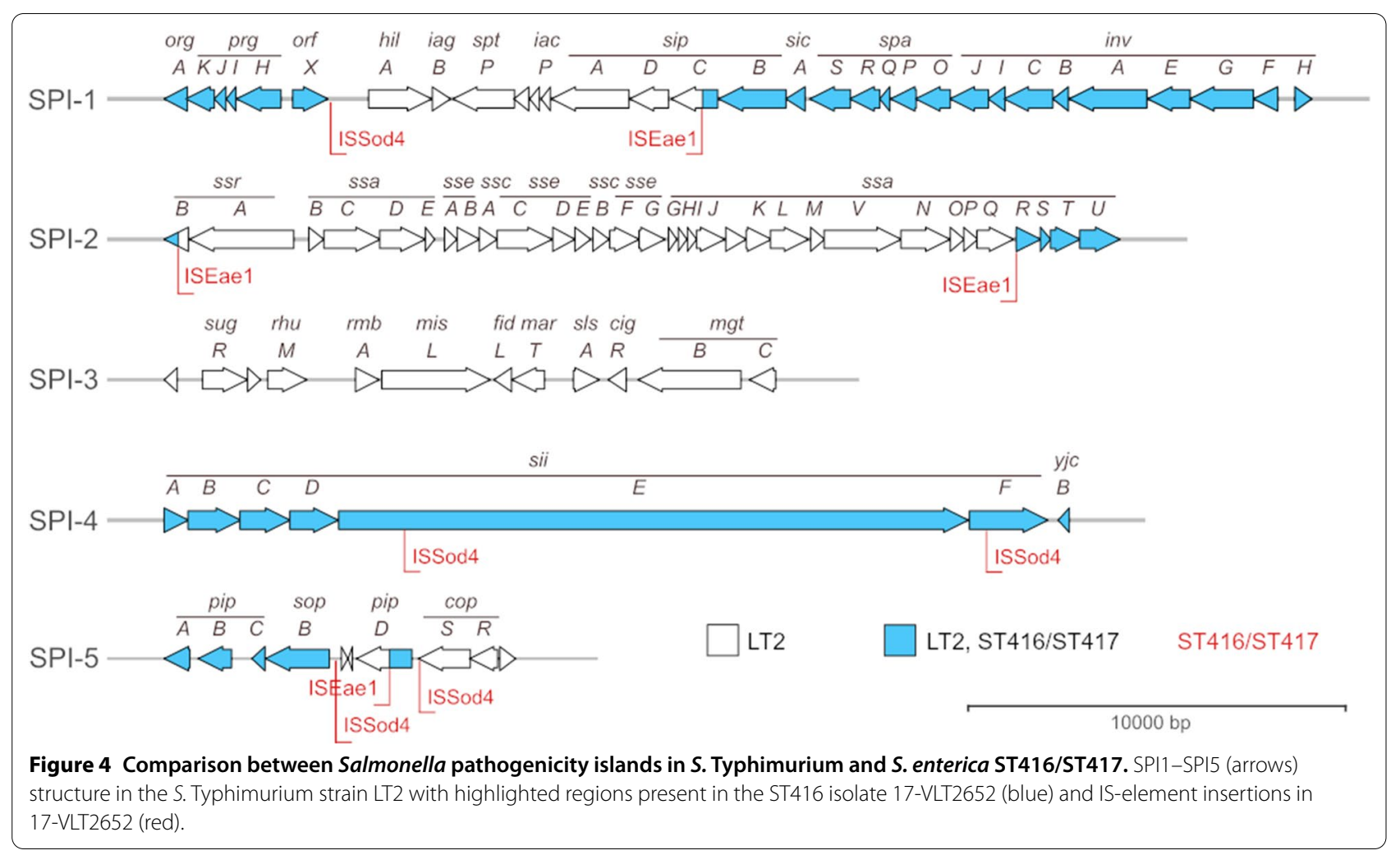

was present elsewhere in the genome flanked by insertion sequence (IS)-element transposase genes. IS256 family ISEae1 or ISSod4 element transposase genes were present flanking the described deletions. The deletions observed in SPI-1, SPI-2, SPI-3 and SPI- 5 were shared among ST416/417 isolates with variation in the extent of the deletions in a few isolates (Additional file 1). In contrast, the elements were mostly conserved in Bispebjerg, Fulica and Abortusequi isolates with only minor variation in SPI-3 and SPI-5 (Additional file 1). Consistent with the changes in SPIs described above, KEGG pathway analysis indicated overrepresentation of alterations in the categories "Salmonella infection", "Bacterial secretion system" and "Bacterial invasion of epithelial cells" (Table 1) in the ST416 isolate 17-VLT002652 compared to the S. enterica type strain LT2.

\section{Alterations in genes encoding metabolic pathways and other biological functions}

ST416/417 isolates were shown by pangenome and SNP analysis to have enriched loss-of-function alterations in several metabolic pathways when compared to LT2 (Table 1). Genes associated with microbial metabolism in diverse environments were particularly strongly affected, as were pathways involved in sensing and responding to environmental cues such as two-component systems and quorum sensing. Contrasting ST416/417 with its close relatives by pangenome analysis revealed that several of the ST416/417 vs. LT2 differences were not shared with the rest of group 4372 (Additional file 2). For example, ST416/417 appear to consistently lack the gene encoding glucarate dehydratase $(g u d D)$ which is part of a locus essential for fermentation of galactarate and glucarate in $S$. Typhimurium. Loss of function in this locus is a trait known to be common only among serovars associated with extraintestinal infection [37]. Several genes of the 4-hydroxyphenylacetate catabolic pathway were missing in ST416/417, consistent with pseudogene formation in this locus in $S$. Typhi [17], as were the formate hydrogenlyase genes $h y c A, B, C, D$ which are active under anaerobic conditions [38]. Also missing was the transcription factor eutR, which is involved in niche recognition and utilization of ethanolamine in the inflamed host intestine by acting as a sensor for this substance [39]. Loss of eutR has previously been linked to host adapted extraintestinal pathovars of Salmonella [18]. Furthermore, multiple genes involved in adhesion, chemotaxis and motility ( $s t d A, B, C ; f l j A ; t s r$, hin) which have been described as frequently disrupted in extraintestinal serovars [18] were absent or significantly altered in ST416/417, as was interestingly the aerotaxis receptor gene aer. 
Table 1 KEGG pathways altered in ST416/ST417

\begin{tabular}{|c|c|c|c|}
\hline Pathway & Pathway name & Pan-genome & SNP \\
\hline stm01100 & Metabolic pathways & 46 & 30 \\
\hline stm01120 & Microbial metabolism in diverse environments & 35 & 9 \\
\hline stm02020 & Two-component system & 20 & 9 \\
\hline stm05132 & Salmonella infection & 13 & 5 \\
\hline stm02060 & Phosphotransferase system (PTS) & 12 & - \\
\hline stm01110 & Biosynthesis of secondary metabolites & 12 & 7 \\
\hline stm03070 & Bacterial secretion system & 12 & - \\
\hline stm00350 & Tyrosine metabolism & 10 & 2 \\
\hline stm00030 & Pentose phosphate pathway & 9 & - \\
\hline stm01220 & Degradation of aromatic compounds & 7 & - \\
\hline stm01200 & Carbon metabolism & 6 & 2 \\
\hline stm02024 & Quorum sensing & 6 & - \\
\hline stm02010 & $A B C$ transporters & 5 & 5 \\
\hline stm00051 & Fructose and mannose metabolism & 5 & 4 \\
\hline stm00520 & Amino sugar and nucleotide sugar metabolism & 5 & 4 \\
\hline stm00910 & Nitrogen metabolism & 5 & - \\
\hline stm00010 & Glycolysis/gluconeogenesis & 5 & - \\
\hline stm01230 & Biosynthesis of amino acids & 4 & - \\
\hline stm00310 & Lysine degradation & 4 & 3 \\
\hline stm05100 & Bacterial invasion of epithelial cells & 3 & - \\
\hline stm00330 & Arginine and proline metabolism & 3 & - \\
\hline stm00630 & Glyoxylate and dicarboxylate metabolism & 3 & 2 \\
\hline stm00650 & Butanoate metabolism & 3 & 2 \\
\hline stm00680 & Methane metabolism & 3 & 2 \\
\hline stm00620 & Pyruvate metabolism & 3 & - \\
\hline stm00260 & Glycine, serine and threonine metabolism & 3 & - \\
\hline stm03430 & Mismatch repair & 3 & - \\
\hline stm00760 & Nicotinate and nicotinamide metabolism & - & 3 \\
\hline stm00250 & Alanine, aspartate and glutamate metabolism & - & 3 \\
\hline stm01503 & Cationic antimicrobial peptide (CAMP) resistance & - & 3 \\
\hline stm03010 & Ribosome & 2 & - \\
\hline stm00071 & Fatty acid degradation & 2 & - \\
\hline stm00400 & Phenylalanine, tyrosine and tryptophan biosynthesis & 2 & - \\
\hline stm00633 & Nitrotoluene degradation & 2 & - \\
\hline stm00640 & Propanoate metabolism & 2 & - \\
\hline stm00500 & Starch and sucrose metabolism & 2 & 4 \\
\hline stm02030 & Bacterial chemotaxis & 2 & - \\
\hline stm00564 & Glycerophospholipid metabolism & 2 & - \\
\hline stm00562 & Inositol phosphate metabolism & 2 & - \\
\hline stm00541 & O-Antigen nucleotide sugar biosynthesis & - & 2 \\
\hline stm00540 & Lipopolysaccharide biosynthesis & - & 2 \\
\hline stm02060 & Phosphotransferase system (PTS) & - & 2 \\
\hline stm00052 & Galactose metabolism & - & 2 \\
\hline stm00230 & Purine metabolism & - & 2 \\
\hline
\end{tabular}

KEGG pathways significantly enriched for presence/absence variation in isolates of ST416/ST417 compared to the S. enterica type strain LT2 as determined by pangenome analysis and identification of high impact (i.e., presumed loss of function) SNPs. 


\section{Acquired accessory genes}

ST416/417 were found to be in possession of a number of genomic regions absent elsewhere in group 4372 (Additional file 2). Several plasmid and prophage-associated genes were included in this unique set, and PlasmidFinder-2 identified three contigs as putative plasmids of incompatibility types IncI1-I(Gamma) (contig 26), IncFIB (contig 3) and IncFII (contig 19). Bandage analysis confirmed that contigs 26 and 19 were indeed consistent with circular plasmids, whereas contig 3 appears to be chromosomal. Notably, the iucA,C,D/iutA aerobactin gene cluster encoding a siderophore iron uptake system associated with extraintestinal infections by both avian pathogenic E. coli (APEC) [40] and poultryassociated Salmonella [41] was uniquely present in all ST416/417. The same was true for the tsh gene encoding a temperature-sensitive hemagglutinin which is also linked to APEC virulence [42], and which was co-located with iucA,C,D/iutA in 17-VLT002652. ST416/417 also uniquely carried a version of the hemR hemin receptor gene, encoding another putative iron uptake system.

\section{Model-based estimation of extraintestinal niche adaptation}

A random forest classifier model previously trained to identify strains associated with extraintestinal disease based on well-characterised gastrointestinal and extraintestinal Salmonella serovars [27] was used to assess all ST416/417 isolates and their closest relatives. The results indicate that all group 4372 have genetic adaptations previously observed in extraintestinal serotypes, with ST416/417 and Abortusequi scoring higher than Bispebjerg and Fulica (Figure 5). However, all tested isolates scored lower than the reference set of extraintestinal serovars.

\section{Discussion}

We here report the first observations of putatively hostadapted group B Salmonella enterica ST416 in harbour porpoises from Swedish waters. Both infected animals were found in a region occupied by porpoises of the Belt Sea population, but genetic analysis would be necessary to confirm their origin. Unlike studies on porpoises from waters around Great Britain where confirmed or presumed ST416/417 have been isolated from 20 to $35 \%$ of porpoises analysed [11, 12, 43], this bacterium was found in only 2 of the 47 lung samples (4\%) examined in this study. However, relatively few porpoises were examined, and isolation rates may have been influenced by isolation protocols, freezing of carcasses prior to examination, and the effects of post-mortem decomposition. The occurrence of closely related strains in Swedish and British

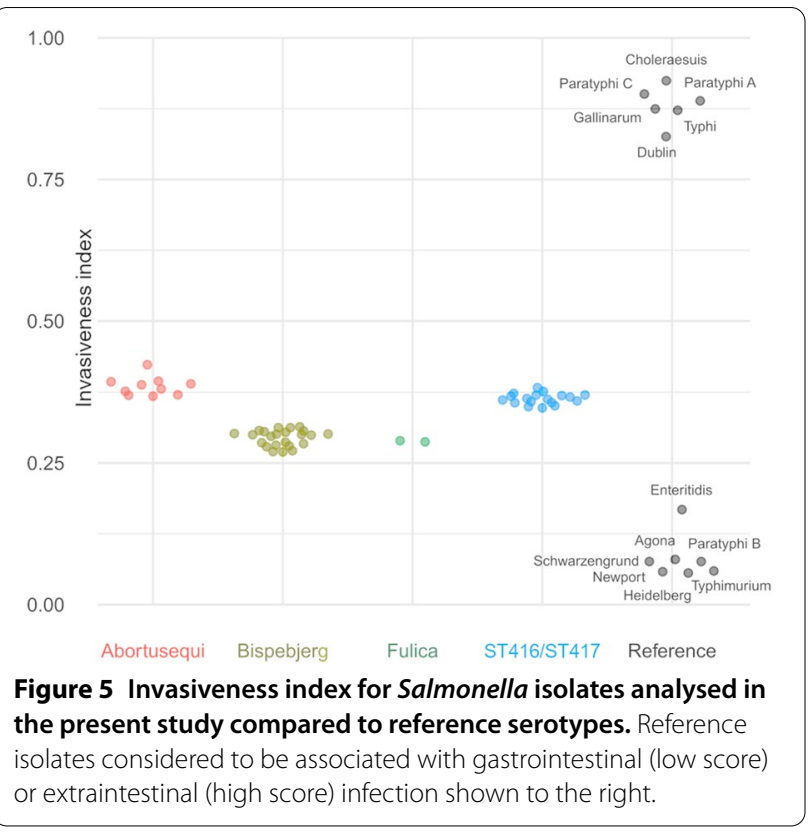

porpoises supports the theory of ST416/417 as specifically adapted for cetacean hosts. To our knowledge and with the exception of one possible case described from the Netherlands [6], host-adapted Salmonella has not been reported in harbour porpoises elsewhere. In a previous study, a few Salmonella sp. isolates were recovered from porpoises in the German Baltic Sea, but no infection was observed in animals from the German North Sea or in the waters around Norway and Greenland [44]. The recovered Salmonella isolates were not typed. Interestingly, Valderrama Vasquez et al. [43] found a significant increase in the frequency of Salmonella infection among porpoises over time from $6 \%$ in $1990-1994$ to $27 \%$ in 2000-2002. The reason for this increase is not known but change in immune competence of the host, for example due to anthropogenic environmental disturbances, cannot be excluded. Further surveillance is needed to better estimate the prevalence of ST416/417 among Swedish porpoises and to monitor changes over time, as well as to investigate possible links between parasite load and risk of salmonellosis for these animals. Recovering more isolates for whole-genome analysis would also be beneficial to determine if ST416/417 has been introduced recently in Swedish waters, as we would expect a long-term established wildlife reservoir to host a higher degree of genetic variation among the bacteria.

Our results show that ST416/417 is not necessarily pathogenic for porpoises, as it was cultured from the lungs of an apparently healthy animal in the absence of associated pathology. However, it did cause serious disease in the other case. This is consistent with findings 
in other studies $[10,11,43]$. These researchers propose that this type of Salmonella is part of the commensal flora in the lung of porpoises but may become an opportunistic pathogen under the right conditions, including immunosuppression. The major genetic virulence factors of S. enterica are encoded on pathogenicity islands, SPIs, and we here show that the key SPIs 1-5 appear to be largely inactivated in ST416/417 in contrast to those of near relatives. These SPIs are believed to have been acquired early in the evolution of S. enterica and most of them are generally considered to be highly conserved among well studied serovars $[15,45]$. It is possible that extensive loss of virulence factors is a common adaptation among commensal and opportunistically pathogenic strains of $S$. enterica, which are less likely to be sampled and sequenced. We note that the inactivation of these elements seems to have been the result of IS element activity, a mechanism of virulence modulation known to occur in a wide range of bacterial species [46].

Gastrointestinal serovars of S. enterica have extensive metabolic capabilities evolved e.g. to exploit nutrients available in the inflamed host gut. The complex genetic network needed for this tends to be degraded in previously studied extraintestinal serovars [17, 18]. Pathway analysis indicates reduced metabolic function in ST416/417, consistent with the fact that this type of Salmonella has in most cases been recovered from extraintestinal samples and in particular from the lungs of infected porpoises. ST416/417 also share other traits common among extraintestinal serovars such as loss of capabilities for sensing and responding to environmental cues. Directly linking alterations contributing to virulence or host adaptation on a gene-by-gene basis is complicated by our limited understanding of the complex networks of gene interaction that determine the fitness of a strain under a particular set of circumstances. Approaches based on machine learning show great promise in solving this type of problem and have recently been applied to Salmonella classification and source attribution [27, 47, 48]. The system implemented in the present study classified ST416/417 isolates as moderately invasive, but the small size and diversity of the training dataset is likely to be a limiting factor. Future improvement of predictions of host bias or disease phenotype based on genomics will strongly depend on the continued collection of high-quality epidemiological and clinical outcome data as well as sequences.

Unsuccessful attempts have been made in the past to find close relatives of the porpoise Salmonella strains using MLST [9], and other molecular methods such as ribotyping, insertion sequence element fingerprinting and PCR-based profiling [49]. Our results using cgMLST largely reflect the results of these previous studies in not being able to find any closely related isolates from other sources, despite the substantial number of genome sequences now available for cgMLST comparison in Enterobase. Again in agreement with results from the aforementioned studies, the closest relatives we do find include some but not all serotypes with similar antigenic profiles to 4,12:a:(Figure 3). Bispebjerg $(1,4,[5], 12: a: e, n, x)$ and the rare Fulica $(4,[5], 12: \mathrm{a}[1,5]:)$ are both poorly characterized and seem to occur in diverse sources including cases of illness in humans. The somewhat less closely related Abortusequi $(4,12:-: \mathrm{e}, \mathrm{n}, \mathrm{x})$ is host restricted to horses [13] and known for causing extraintestinal infections in the form of equine paratyphoid characterized by abortions in mares and septicaemia in young foals [50]. Interestingly, Abortusequi has been recovered in high numbers from parasitic aneurysms in apparently healthy and otherwise Salmonella-negative horses [51], with the hypothesis raised that the lesions provided a niche for long-term carriage of the bacteria. An outlier without serotype assignment among the identified related strains was recovered by metagenomic sequencing from the tooth pulp of a Neolithic hunter-gatherer, presumably suffering from systemic infection [52]. In general, a parallel can perhaps be drawn between this lineage of S. enterica and that containing the generalist serovar Enteritidis, the host-adapted Dublin (cattle) and the host-restricted Gallinarum (poultry) [15] with a set of shared adaptations providing evolutionary opportunities for further specialization for different hosts. We intend to further investigate this by targeted sequencing of historical Salmonella isolates with antigen profiles similar to 4,12:a:- from other wildlife sources.

As previously mentioned, further studies will also be necessary to determine the relevance of extraintestinal salmonellosis as a health threat for porpoises and the possible emergent health effect of the combination of microbial infections, parasite load and environmental stressors. While the public health relevance of porpoise Salmonella strains is likely to be low due to limited exposure and likely reduced virulence, other host adapted serovars of Salmonella including Dublin [53], Choleraesuis [54], and certain Typhimurium [55] commonly cause illness in humans. Adaptation for causing extraintestinal infection in some of these serovars in fact increases the risk of severe and life-threatening systemic Salmonella infections, generally affecting the elderly or otherwise vulnerable [53, 54]. A better understanding of the genetic changes underlying the evolution of host adaptation and extraintestinal niche specialization therefore has the potential to be highly valuable both for improving animal and human health. 


\section{Abbreviations}

bp: base pairs; BG agar: brilliant green agar; CgMLST: core genome multi-locus sequence typing; IS: insertion sequence; MALDI-TOFMS: matrix assisted laser desorption ionization-time of flight mass spectrometry; MLST: multi-locus sequence typing; MSRV agar: modified semi-solid Rappaport-Vassiliadis agar; PCR: polymerase chain reaction; SNP: single nucleotide polymorphism; SPI: Salmonella Pathogenicity island; ST: sequence type; SVA: Swedish National Veterinary Institute; T1SS/T3SS: type 1/type 3 secretion system; XLD agar: xylose lysine deoxycholate agar.

\section{Supplementary Information}

The online version contains supplementary material available at https://doi. org/10.1186/s13567-021-01001-0.

\section{Additional file 1. Deletions in Salmonella pathogenicity islands in} S. enterica ST416/ST417. Presence (black) or absence (grey) of genes in Salmonella pathogenicity islands SPI-1,2,3,5 as determined by pangenome (Roary) analysis. The two Swedish isolates from the present study are labelled SE. Extensive deletions are evident in all ST416/ST417 isolates, while the islands are highly conserved among other related strains.

Additional file 2. Pan-genome analysis output, presence/absence and significance testing. Number of isolates of ST416/ST417 (referred to as "positive") and other related Salmonella isolates (referred to as "negative") that were found by pan-genome analysis to posess each identified sequence, i.e. gene or gene variant, and significance testing results for comparison of the frequence of occurrence in the two groups.

Additional file 3. Pan-genome analysis output, FASTA reference sequences. FASTA format sequences for the "groups" of sequence variants compared in Additional file 2.

Additional file 4. $\mathrm{R}$ script for processing SNP data. A short script for the $R$ software environment used to process and filter SNP data.

\section{Acknowledgements}

The authors would like to thank Erik Ågren, Tobias Lilja and the SVA Salmonella laboratory for technical assistance.

\section{Authors' contributions}

AN and AR secured funding, organized the collection of animals and performed and interpreted post-mortem examinations. JE performed and interpreted microbiological analyses. AKSS and RS organized sequencing, performed the bioinformatic analyses and wrote the first manuscript draft. All authors read and approved the final manuscript.

\section{Funding}

Funding support for the collection and examination of harbour porpoises was provided by The Swedish Agency for Marine and Water Management and The Swedish Environmental Protection Agency. Funders had no influence over how the study was performed, the manuscript text or the decision to publish.

\section{Availability of data and materials}

Sequence data from long and short read sequencing with metadata have been deposited in the European Nucleotide Archive [56] under project accession number PRJEB40444. Typing data from cgMLST analysis has been deposited in Enterobase under isolate IDs (uberstrains) SAL_EB4912AA and SAL_EB4913AA. Pan-genome analysis output is presented in Additional files 2 and 3 .

\section{Declarations}

Ethics approval and consent to participate

All animals were examined post-mortem as part of routine national wildlife surveillance. Therefore, this study required no ethics approval by Swedish law.

\section{Competing interests}

The authors declare that they have no competing interests.

\section{Author details}

${ }^{1}$ Department of Microbiology, National Veterinary Institute, Uppsala, Sweden. ${ }^{2}$ Department of Pathology and Wildlife Diseases, National Veterinary Institute, Uppsala, Sweden. ${ }^{3}$ Department of Environmental Research and Monitoring, Swedish Museum of Natural History, Stockholm, Sweden.

Received: 19 May 2021 Accepted: 21 September 2021 Published online: 21 October 2021

References

1. Braulik GM, Minton G, Amano M, Bjørge A (2020) Phocoena phocoena. The IUCN Red List of Threatened Species 2020. IUCN

2. Van Bressem MF, Raga JA, Di Guardo G, Jepson PD, Duignan PJ, Siebert U, Barrett T, Santos MC, Moreno IB, Siciliano S, Aguilar A, Van Waerebeek $K$ (2009) Emerging infectious diseases in cetaceans worldwide and the possible role of environmental stressors. Dis Aquat Organ 86:143-157. https://doi.org/10.3354/dao02101

3. Neimane A, Stavenow J, Ågren E, Wikström E, Roos A (2020) Hälso-och sjukdomsövervakning av marina däggdjur Del 2. Hälsa, sjukdomar och dödsorsaker hos tumlare (Phocoena phocoena) i Sverige de senaste 10 åren. National Veterinary Institute (SVA), Sweden

4. Jauniaux T, Petitjean D, Brenez C, Borrens M, Brosens L, Haelters J, Tavernier T, Coignoul F (2002) Post-mortem findings and causes of death of harbour porpoises (Phocoena phocoena) stranded from 1990 to 2000 along the coastlines of Belgium and Northern France. J Comp Pathol 126:243-253. https://doi.org/10.1053/jcpa.2001.0547

5. Jepson PD, Baker JR, Kuiken T, Simpson VR, Kennedy S, Bennett PM (2000) Pulmonary pathology of harbour porpoises (Phocoena phocoena) stranded in England and Wales between 1990 and 1996. Vet Rec 146:721-728. https://doi.org/10.1136/vr.146.25.721

6. van Elk CE, van de Bildt MWG, van Run P, Bunskoek P, Meerbeek J, Foster G, Osterhaus A, Kuiken T (2019) Clinical, pathological, and laboratory diagnoses of diseases of harbour porpoises (Phocoena phocoena), live stranded on the Dutch and adjacent coasts from 2003 to 2016. Vet Res 50:88. https://doi.org/10.1186/s13567-019-0706-3

7. Fenton H, Daoust PY, Forzan MJ, Vanderstichel RV, Ford JK, Spaven L, Lair S, Raverty S (2017) Causes of mortality of harbor porpoises Phocoena phocoena along the Atlantic and Pacific coasts of Canada. Dis Aquat Organ 122:171-183. https://doi.org/10.3354/dao03080

8. Reckendorf A, Everaarts E, Bunskoek P, Haulena M, Springer A, Lehnert K, Lakemeyer J, Siebert U, Strube C (2021) Lungworm infections in harbour porpoises (Phocoena phocoena) in the German Wadden Sea between 2006 and 2018, and serodiagnostic tests. Int J Parasitol Parasites Wildl 14:53-61. https://doi.org/10.1016/j.ijppaw.2021.01.001

9. Haase JK, Brown DJ, Weill FX, Mather H, Foster G, Brisse S, Wain J, Achtman M (2012) Population genetic structure of 4,12:a:- Salmonella enterica strains from harbor porpoises. Appl Environ Microbiol 78:8829-8833. https://doi.org/10.1128/AEM.02310-12

10. Davison NJ, Simpson VR, Chappell S, Monies RJ, Stubberfield EJ, Koylass M, Quinney S, Deaville R, Whatmore AM, Jepson PD (2010) Prevalence of a host-adapted group B Salmonella enterica in harbour porpoises (Phocoena phocoena) from the south-west coast of England. Vet Rec 167:173-176. https://doi.org/10.1136/vr.c3760

11. Foster G, Patterson IA, Munro DS (1999) Monophasic group B Salmonella species infecting harbour porpoises (Phocoena phocoena) inhabiting Scottish coastal waters. Vet Microbiol 65:227-231. https://doi.org/10. 1016/s0378-1135(98)00296-x

12. Davison N, Barnett J, Rule B, Chappell S, Wise G (2010) Group B Salmonella in lungworms from a harbour porpoise (Phocoena phocoena). Vet Rec 167:351-352. https://doi.org/10.1136/vr.c4495

13. Uzzau S, Brown DJ, Wallis T, Rubino S, Leori G, Bernard S, Casadesus J, Platt DJ, Olsen JE (2000) Host adapted serotypes of Salmonella enterica. Epidemiol Infect 125:229-255. https://doi.org/10.1017/s09502688990043 79

14. Parkhill J, Dougan G, James KD, Thomson NR, Pickard D, Wain J, Churcher C, Mungall KL, Bentley SD, Holden MT, Sebaihia M, Baker S, Basham D, Brooks K, Chillingworth T, Connerton P, Cronin A, Davis P, Davies RM, Dowd L, White N, Farrar J, Feltwell T, Hamlin N, Haque A, Hien TT, Holroyd S, Jagels K, Krogh A, Larsen TS, Leather S, Moule S, O'Gaora P, Parry C, 
Quail M, Rutherford K, Simmonds M, Skelton J, Stevens K, Whitehead S, Barrell BG (2001) Complete genome sequence of a multiple drug resistant Salmonella enterica serovar Typhi CT18. Nature 413:848-852. https:// doi.org/10.1038/35101607

15. Langridge GC, Fookes M, Connor TR, Feltwell T, Feasey N, Parsons BN, Seth-Smith HM, Barquist L, Stedman A, Humphrey T, Wigley P, Peters SE, Maskell DJ, Corander J, Chabalgoity JA, Barrow P, Parkhill J, Dougan G, Thomson NR (2015) Patterns of genome evolution that have accompanied host adaptation in Salmonella. Proc Natl Acad Sci USA 112:863-868. https://doi.org/10.1073/pnas.1416707112

16. Chiu CH, Tang P, Chu C, Hu S, Bao Q, Yu J, Chou YY, Wang HS, Lee YS (2005) The genome sequence of Salmonella enterica serovar Choleraesuis, a highly invasive and resistant zoonotic pathogen. Nucleic Acids Res 33:1690-1698. https://doi.org/10.1093/nar/gki297

17. Seif Y, Kavvas E, Lachance JC, Yurkovich JT, Nuccio SP, Fang X, Catoiu E, Raffatellu M, Palsson BO, Monk JM (2018) Genome-scale metabolic reconstructions of multiple Salmonella strains reveal serovar-specific metabolic traits. Nat Commun 9:3771. https://doi.org/10.1038/s41467-018-06112-5

18. Nuccio SP, Baumler AJ (2014) Comparative analysis of Salmonella genomes identifies a metabolic network for escalating growth in the inflamed gut. MBio 5:e00929-14. https://doi.org/10.1128/mBio.00929-14

19. IJsseldijk LL, Brownlow AC, Mazzariol S (2019) Best practice on cetacean post mortem investigation and tissue sampling. Joint ACCOBAMS and ASCOBANS document

20. Bancroft JD, Cook HC (1984) Manual of histological techniques. Churchill Livingstone, London

21. Hoorfar J, Ahrens P, Radstrom P (2000) Automated $5^{\prime}$ nuclease PCR assay for identification of Salmonella enterica. J Clin Microbiol 38:3429-3435. https://doi.org/10.1128/JCM.38.9.3429-3435.2000

22. Enterobase. https://enterobase.warwick.ac.uk/

23. De Coster W, D'Hert S, Schultz DT, Cruts M, Van Broeckhoven C (2018) NanoPack: visualizing and processing long-read sequencing data. Bioinformatics 34:2666-2669. https://doi.org/10.1093/bioinformatics/bty149

24. Cingolani P, Platts A, le Wang L, Coon M, Nguyen T, Wang L, Land SJ, Lu X, Ruden DM (2012) A program for annotating and predicting the effects of single nucleotide polymorphisms, SnpEff: SNPs in the genome of Drosophila melanogaster strain w1118; iso-2; iso-3. Fly 6:80-92. https://doi.org/ 10.4161/fly.19695

25. Mi H, Muruganujan A, Casagrande JT, Thomas PD (2013) Large-scale gene function analysis with the PANTHER classification system. Nat Protoc 8:1551-1566. https://doi.org/10.1038/nprot.2013.092

26. Kanehisa M, Sato Y (2020) KEGG Mapper for inferring cellular functions from protein sequences. Protein Sci 29:28-35. https://doi.org/10.1002/ pro. 3711

27. Wheeler NE, Gardner PP, Barquist L (2018) Machine learning identifies signatures of host adaptation in the bacterial pathogen Salmonella enterica. PLoS Genet 14:e1007333. https://doi.org/10.1371/journal.pgen.1007333

28. Wick RR, Judd LM, Gorrie CL, Holt KE (2017) Unicycler: resolving bacterial genome assemblies from short and long sequencing reads. PLoS Comput Biol 13:e1005595. https://doi.org/10.1371/journal.pcbi.1005595

29. Wick RR, Schultz MB, Zobel J, Holt KE (2015) Bandage: interactive visualization of de novo genome assemblies. Bioinformatics 31:3350-3352. https://doi.org/10.1093/bioinformatics/btv383

30. Seemann T (2014) Prokka: rapid prokaryotic genome annotation. Bioinformatics 30:2068-2069. https://doi.org/10.1093/bioinformatics/btu153

31. Page AJ, Cummins CA, Hunt M, Wong VK, Reuter S, Holden MT, Fookes M, Falush D, Keane JA, Parkhill J (2015) Roary: rapid large-scale prokaryote pan genome analysis. Bioinformatics 31:3691-3693. https://doi.org/10. 1093/bioinformatics/btv421

32. Brynildsrud O, Bohlin J, Scheffer L, Eldholm V (2016) Rapid scoring of genes in microbial pan-genome-wide association studies with Scoary. Genome Biol 17:238. https://doi.org/10.1186/s13059-016-1108-8

33. The R project for statistical computing. https://www.r-project.org/

34. gggenes. https://wilkox.org/gggenes/

35. Silva C, Puente JL, Calva E (2017) Salmonella virulence plasmid: pathogenesis and ecology. Pathog Dis 75:ftx070. https://doi.org/10.1093/femspd/ $\mathrm{ft} \times 070$

36. Lou L, Zhang P, Piao R, Wang Y (2019) Salmonella pathogenicity island 1 (SPI-1) and its complex regulatory network. Front Cell Infect Microbiol 9:270. https://doi.org/10.3389/fcimb.2019.00270
37. Faber F, Tran L, Byndloss MX, Lopez CA, Velazquez EM, Kerrinnes T, Nuccio SP, Wangdi T, Fiehn O, Tsolis RM, Baumler AJ (2016) Host-mediated sugar oxidation promotes post-antibiotic pathogen expansion. Nature 534:697-699. https://doi.org/10.1038/nature18597

38. Lamichhane-Khadka R, Benoit SL, Miller-Parks EF, Maier RJ (2015) Host hydrogen rather than that produced by the pathogen is important for Salmonella enterica serovar Typhimurium virulence. Infect Immun 83:311-316. https://doi.org/10.1128/IAI.02611-14

39. Anderson CJ, Clark DE, Adli M, Kendall MM (2015) Ethanolamine signaling promotes Salmonella niche recognition and adaptation during infection. PLoS Pathog 11:e1005278. https://doi.org/10.1371/journal.ppat.1005278

40. Gao Q, Wang X, Xu H, Xu Y, Ling J, Zhang D, Gao S, Liu X (2012) Roles of iron acquisition systems in virulence of extraintestinal pathogenic Escherichia coli: salmochelin and aerobactin contribute more to virulence than heme in a chicken infection model. BMC Microbiol 12:143. https:// doi.org/10.1186/1471-2180-12-143

41. Wellawa DH, Allan B, White AP, Koster W (2020) Iron-uptake systems of chicken-associated Salmonella serovars and their role in colonizing the avian host. Microorganisms 8:1203. https://doi.org/10.3390/microorgan isms 8081203

42. Dozois CM, Dho-Moulin M, Bree A, Fairbrother JM, Desautels C, Curtiss R 3rd (2000) Relationship between the Tsh autotransporter and pathogenicity of avian Escherichia coli and localization and analysis of the tsh genetic region. Infect Immun 68:4145-4154. https://doi.org/10.1128/iai. 68.7.4145-4154.2000

43. Valderrama Vasquez CA, Macgregor SK, Rowcliffe JM, Jepson PD (2008) Occurrence of a monophasic strain of Salmonella group B isolated from cetaceans in England and Wales between 1990 and 2002. Environ Microbiol 10:2462-2468. https://doi.org/10.1111/j.1462-2920.2008.01651.x

44. Siebert U, Prenger-Berninghoff E, Weiss R (2009) Regional differences in bacterial flora in harbour porpoises from the North Atlantic: environmental effects? J Appl Microbiol 106:329-337. https://doi.org/10.1111/j.13652672.2008.04006.x

45. Hensel M (2004) Evolution of pathogenicity islands of Salmonella enterica. Int J Med Microbiol 294:95-102. https://doi.org/10.1016/j.jmm.2004.06. 025

46. Vandecraen J, Chandler M, Aertsen A, Van Houdt R (2017) The impact of insertion sequences on bacterial genome plasticity and adaptability. Crit Rev Microbiol 43:709-730. https://doi.org/10.1080/1040841X.2017.13036 61

47. Lupolova N, Lycett SJ, Gally DL (2019) A guide to machine learning for bacterial host attribution using genome sequence data. Microb Genom 5:e000317. https://doi.org/10.1099/mgen.0.000317

48. Zhang S, Li S, Gu W, den Bakker H, Boxrud D, Taylor A, Roe C, Driebe E, Engelthaler DM, Allard M, Brown E, McDermott P, Zhao S, Bruce BB, Trees E, Fields PI, Deng X (2019) Zoonotic source attribution of Salmonella enterica serotype Typhimurium using genomic surveillance data, United States. Emerg Infect Dis 25:82-91. https://doi.org/10.3201/eid2501. 180835

49. Old DC, Crichton PB, Taylor A, Mather H (2001) An attempt to identify the evolutionary origin of a novel serotype of Salmonella enterica isolated from harbour porpoises. J Med Microbiol 50:415-420. https://doi.org/10. 1099/0022-1317-50-5-415

50. Grandolfo E, Parisi A, Ricci A, Lorusso E, de Siena R, Trotta A, Buonavoglia D, Martella V, Corrente M (2018) High mortality in foals associated with Salmonella enterica subsp. enterica Abortusequi infection in Italy. J Vet Diagn Invest 30:483-485. https://doi.org/10.1177/1040638717753965

51. Niwa H, Hobo S, Kinoshita Y, Muranaka M, Ochi A, Ueno T, Oku K, Hariu K, Katayama Y (2016) Aneurysm of the cranial mesenteric artery as a site of carriage of Salmonella enterica subsp. enterica serovar Abortusequi in the horse. J Vet Diagn Invest 28:440-444. https://doi.org/10.1177/10406 38716649640

52. Key FM, Posth C, Esquivel-Gomez LR, Hubler R, Spyrou MA, Neumann GU, Furtwangler A, Sabin S, Burri M, Wissgott A, Lankapalli AK, Vagene AJ, Meyer M, Nagel S, Tukhbatova R, Khokhlov A, Chizhevsky A, Hansen S, Belinsky AB, Kalmykov A, Kantorovich AR, Maslov VE, Stockhammer PW, Vai S, Zavattaro M, Riga A, Caramelli D, Skeates R, Beckett J, Gradoli MG, Steuri N, Hafner A, Ramstein M, Siebke I, Losch S, Erdal YS, Alikhan NF, Zhou Z, Achtman M, Bos K, Reinhold S, Haak W, Kuhnert D, Herbig A, Krause J (2020) Emergence of human-adapted Salmonella enterica is 
linked to the Neolithization process. Nat Ecol Evol 4:324-333. https://doi. org/10.1038/s41559-020-1106-9

53. Harvey RR, Friedman CR, Crim SM, Judd M, Barrett KA, Tolar B, Folster JP, Griffin PM, Brown AC (2017) Epidemiology of Salmonella enterica serotype Dublin infections among humans, United States, 1968-2013. Emerg Infect Dis 23:1493-1501. https://doi.org/10.3201/eid2309.170136

54. Chiu CH, Su LH, Chu C (2004) Salmonella enterica serotype Choleraesuis: epidemiology, pathogenesis, clinical disease, and treatment. Clin Microbiol Rev 17:311-322. https://doi.org/10.1128/cmr.17.2.311-322.2004

55. Soderlund R, Jernberg C, Tronnberg L, Paajarvi A, Agren E, Lahti E (2019) Linked seasonal outbreaks of Salmonella Typhimurium among passerine birds, domestic cats and humans, Sweden, 2009 to 2016. Euro Surveill 24:1900074. https://doi.org/10.2807/1560-7917.ES.2019.24.34.1900074

56. European nucleotide archive. https://www.ebi.ac.uk/ena

\section{Publisher's Note}

Springer Nature remains neutral with regard to jurisdictional claims in published maps and institutional affiliations.
Ready to submit your research? Choose BMC and benefit from:

- fast, convenient online submission

- thorough peer review by experienced researchers in your field

- rapid publication on acceptance

- support for research data, including large and complex data types

- gold Open Access which fosters wider collaboration and increased citations

- maximum visibility for your research: over $100 \mathrm{M}$ website views per year

At BMC, research is always in progress.

Learn more biomedcentral.com/submissions 